bei den Samen der einzelnen Bäume sehr unterschiedlich, ohne da $\beta$ ein Einflu $\beta$ des Standorts festgestellt werden konnte. Zur Veranschaulichung wurden für das Histogramm (Fig. 2) als Beispiel 4 Bäume herausgegriffen. Andere Untersuchungen, ob zwischen dem Saugkraftvermögen von Kiefersamen und der Diirreresistenz im Keimlingsstadium eine Korrelation besteht, werden zur Zeit durchgeführt.

Forstbotanisches Institut der Fakultät fïr Forstwirtschaft. Tharandt

Eingegangen am 12. Mai 1960

WERNER ZENTSCH

1) GASSNer, G., u. E. BatmGarten: Züchter 22, H. 3, 65 (1952).

\section{Hemixis in Blepharisma Undulans (Ciliophora: Spirotricha)}

DILLER ${ }^{I}$ ) reported the existence of 'Hemixis' - a process involving macronuclear behavior in the mass cultures of Paramecium aurelia and $P$. caudatum. This is a process of macronuclear reorganisation in which the macronucleus undergoes a series of autonomous changes during vegetative life exclusive of those of normal binary fission. Hemixis is primarily a process of macronuclear fragmentation and division without any micronuclear activity.

Blepharisma undulans used in the present study was obtained from the original stock cultures maintained in this laboratory for a number of years. It differs from the races described by YounG ${ }^{2}$ ), WeIsz ${ }^{3}$ ) and Suzouki ${ }^{4}$ ) principally in the structure of the macronucleus ${ }^{5}$ ). There are 4 types of hemixis observed in these forms:

Type $A$, is the simplest form of hemixis, characterized by a precocious division of the macronucleus into two or more parts. This đivision is not synchronized with the micronuclear division. There appear clear reorganisation bands dividing the macronucleus into two or three parts.

Type $B$, considered to be common, is characterized by the resolution of the macronucleus into spherical bodies of varying sizes which disintegrate in the cytoplasm.

Type $C$, is characterized by splitting of the macronucleus into two or more major portions giving an appearance of a branched structure like the skeining during conjugation. This is followed by extrusion of the macronuclear material into the cytoplasm.

Type $D$, is considered to be pathological condition where in the macronucleus undergoes complete dissolution. Micronuclei generally disappear before the dissolution of the macronucleus.

I am greatly indebted to Prof. B. R. Seshachar, D. Sc., for guidance and encouragement.

Dept. of Zoology, Central College, Bangalove (India)

Eingegangen am 23. Mai 1960

(Miss) P.B. Padmavathi

1) DILLER, W.F.: J. Morphology 59, 11 (1936). - 2) Young, D.: J. Morphology 64, 297 (1939). - 3) WEISz, P.B.: J. Morphology 85, 503 (1949). - ${ }^{4}$ ) Suzoukr, Y.: J. Sci. Hiroshima Univ. 15, 205 (1951). - 5) Padmavathi, P.B.: Ves. Csl. Zool. Spolec. 23, 193 (1959).

Innersekretorische Wirkung auf Strukturmodifikationen der Speicheldrüsenchromosomen von Acricotopus lucidus (Chironomide)

In den Lappen der Speicheldrüse von $A$. lucidus bilden bestimmte Chromosomenloci Balbiani-Ringe, die sowohl lappen- wie stadienspezifisch sind ${ }^{1}$ ). Solche Strukturmodifikationen werden nach. BEERMANN als Ausdruck differentieller Genaktivität gedeutet $2 a, b)$. Im Rahmen eines von MEchelKe aufgestellten Programms zur Erforschung der Einflüsse innerer und äußerer Faktoren auf die physiologische Aktivität bestimmter Chromosomenloci bei A. lucidus wurde die Frage geprüft, ob eine Beziehung zwischen dem innersekretorischen Milieu und den Balbiani-Ringen besteht.

Bei normaler Entwicklung vom letzten Larven- zum Vorpuppenstadium bleiben die für den Haupt- und Nebenlappen spezifischen Balbiani-Ringe $\mathrm{BR}_{1}$ und $\mathrm{BR}_{2}$ unverändert, während die für den Vorderlappen spezifischen Balbiani-Ringe $\mathrm{BR}_{3}$ und $\mathrm{BR}_{4}$ rückgebildet werden. Speicheldrüsen, in denen alle 4 Balbiani-Ringe voll ausgebildet waren, wurden 12 bis 48 Std bei $+10^{\circ} \mathrm{C}$ in vitro unter dem Einflü folgender Lymphebedingungen gehalten: 1. larvale, körpereigene Lymphe; 2. präpupale Lymphe kombiniert mit pupaler Lymphe; 3. larvale Lymphe kombiniert mit Gehirnkomplex junger Puppen, bestehend aus Ober- und Unterschlundganglion mit Anhangdrüsen. Dabei zeigten im 1. Fall alle Balbiani-Ringe keine Veränderung ihrer Grundstruktur. Im 2. Fall blieb die Grundstruktur der Balbiani-Ringe $\mathrm{BR}_{1}$ und $\mathrm{BR}_{\mathrm{g}}$ bestehen, während die Balbiani-Ringe $B_{3}$ und $B R_{4}$ rückgebildet wurden. Die gieichen Befunde ergaben sich auch im 3. Fall, jedoch bereits nach kürzerer Zeit.

Daraus wird gefolgert, daß die Rückbildung der BalbianiRinge $\mathrm{BR}_{3}$ und $\mathrm{BR}_{4}$ nicht autonom erfolgt, sondern dem Einfluß innersekretorischer Faktoren unterliegt.

Institut für Kulturptlanzenforschung der Deutschen Akademie der Wissenschaften zu Berlin, Gatersleben (Krs. Aschersleben)

Eingegangen am 2. Juni 1960

R. PANITZ

1) Mechelke, F.: Chromosoma 5, 511 (1953). - 2) Bemranan, W.: a) Z. Naturforsch. 7b, 237 (1952); b) Chromosoma 5, 139 (1952).

\section{Über die Beziehung des Plexus des dritten Ventrikels} zum subfornikalen Organ bei den Primaten

Das bei allen höheren Wirbeltieren nachgewiesene subfornikale Organ bildet bei den Säugetieren zusammen mit der Area postrema innerhalb der circumventrikulären Organe ${ }^{3 b}$ ) eine Gruppe ${ }^{14}$ ), die im Gegensatz $z u$ fast allen anderen circumventrikulären Organen in ihrer typischen Differenzierung nur einen Teil der Hirnwand umfaßt. Die in gewissen Grenzen artverschiedene Topik des Sfo drückt sich in seiner Bezeichnung bei den unabhängig voneinander erfolgten Erstbeschreibungen aus, indem es Ganglion psalterii ${ }^{11}$ ), intercolumnar tubercle ${ }^{7}$ ) und subfornikales Organ ${ }^{6}$ genannt wird in $\mathrm{Ab}$ hängigkeit von seiner Lage bei den jeweils vorzugsweise untersuchten Spezies [WATERMANN ${ }^{13}$ a), ${ }^{14}$ ) schlägt mit Rücksicht auf seine Verbreitung bei niederen Vertebraten Interventrikularorgan vor]. Bei fast allen Autoren findet sich übereinstimmend der Hinweis, daB der Plexus des 3. Ventrikels lateral, caudal oder auf dem Sfo ansetze, es also weitgehend bedecke, ohne daß diese Beobachtungen jemals konsequent weiter verfolgt worden wären.

Bei Untersuchung eines größeren Primatenmaterials $\left.{ }^{3}\right)^{12}$ ) bot sich diese Lagebezichung bei Aotes trivirgatus und ähnlich bei Galago crassicaudatus in besonders eindrucksvoller Weise dar und lieferte neue Aspekte für die Stellung des Sfo innerhalb der circumventrikulären Organe.

So dringt bei Aotes die ,,Tela chlorioidea superior"15) in sagittaler Richtung bis über seine höchste Erhebung hinaus in das Sfo vor. (Nach der üblichen Terminologie hieße dies, der Plexus setzte auf dem Sfo an.) Von der Gehirnoberfläche aus betrachtet, liegt hier in der Medianen der am weitesten nasal gelegene Punkt der Fissura transversa, ausgefüllt von der Cisterna fissurae transversae $=$ Cisterna ambiens $^{9}$ ), zwischen Hirnstamm, Kleinhirn- und Großhirnhemisphären gelegen. Die die Fissura ausfüllende Tela besteht auch innerhalb des Sfo aus dem Gerüstwerk des subarachnoidealen Bindegewebes, oberflächlich mit einer Pialamelle abgeschlossen und zahlreiche Gefäße enthaltend, die ventrikelwärts vielfach gewunden und zum Teil Schlingen bildend in das Sfo eindringen. - Sein übriges Gefäßnetz ist dagegen ausgesprochen locker. - Solche Arteriolen sind von einer starken adventitiellen Hülle begleitet, von der aus Bindegewebsfasern tief in das Organ eindringen.

Die durch die Tela abgegliederte ventrikuläre innere Zone des Sfo besteht überwiegend aus dichtgepackten makrogliösen Elementen, die am ehesten mit Ependymzellen verglichen werden können. Daneben kommen vereinzelt ,,kleine Parenchymzellen "1) vor. Diese Innenzone setzt sich scharf von der caudal und lateral sich fortsetzenden plexustragenden Tela $a b$ und ist außerdem durch nur hier lateral auftretende riesige interstitielle Lücken $\left.\left.{ }^{2}\right), 3 \mathrm{~b}\right), 12$ ), $13 \mathrm{~b}$ ) als Bestandteil des Sfo gekennzeichnet. Diese Zone zeigt sehr starke Ähnlichkeit mit dem GefäBorgan der Lamina terminalis ${ }^{3}$ ),${ }^{4}$ ).

Das Sfo bei Aotes besteht demnach aus einem dem Corpus fornicis unmittelbar aufsitzenden Körper und in seiner caudalen Häfte außerdem noch aus einer vielschichtigen gefäß- und bindegewebsreichen ventrikulären Zone, genetisch ein Bestand teil des ursprünglich epithelialen Daches des 3. Ventrikels. Da sich zwischen Körper und Innenzone der mediane Fortsatz der Cisterna ambiens schiebt, grenzt das Organ nicht nur an den 3. Ventrikel, sondern besitzt auch eine mindestens ebensogroße Oberfläche gegenüber den äußeren Liquorräumen des Gehirns. 